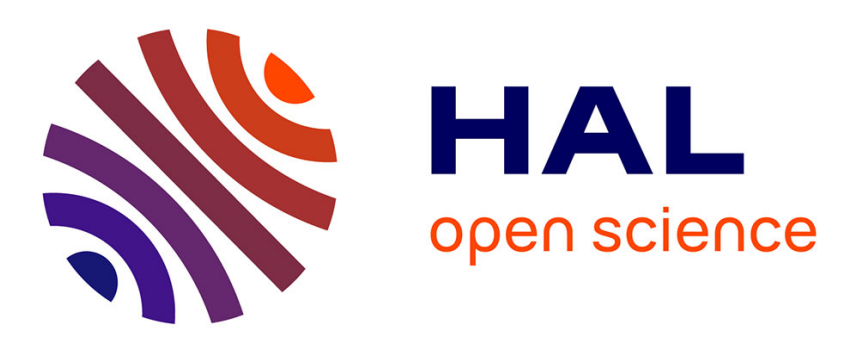

\title{
A new lower bound on the maximum correlation of a set with mismatched filters
}

\author{
Uy Hour Tan, Fabien Arlery, Olivier Rabaste, Frederic Lehmann, \\ Jean-Philippe Ovarlez
}

\section{- To cite this version:}

Uy Hour Tan, Fabien Arlery, Olivier Rabaste, Frederic Lehmann, Jean-Philippe Ovarlez. A new lower bound on the maximum correlation of a set with mismatched filters. IEEE Transactions on Information Theory, 2020, 66 (10), pp.6555-6565. 10.1109/TIT.2020.3002066 . hal-02962309

\section{HAL Id: hal-02962309 \\ https://hal.science/hal-02962309}

Submitted on 14 Jan 2021

HAL is a multi-disciplinary open access archive for the deposit and dissemination of scientific research documents, whether they are published or not. The documents may come from teaching and research institutions in France or abroad, or from public or private research centers.
L'archive ouverte pluridisciplinaire HAL, est destinée au dépôt et à la diffusion de documents scientifiques de niveau recherche, publiés ou non, émanant des établissements d'enseignement et de recherche français ou étrangers, des laboratoires publics ou privés. 


\title{
A New Lower Bound on the Maximum Correlation of a Set with Mismatched Filters
}

\author{
Uy Hour Tan*, Fabien Arlery ${ }^{\dagger}$, Olivier Rabaste ${ }^{\ddagger}$, Frédéric Lehmann ${ }^{\S}$ and Jean-Philippe Ovarlez ${ }^{\ddagger}$ \\ * Thales Land \& Air Systems, Élancourt, France \\ $\dagger$ Thales Defense Mission Systems, Élancourt, France \\ $\ddagger$ DEMR, ONERA, Université Paris-Saclay, Palaiseau, France \\ $\S$ SAMOVAR, Telecom SudParis, CNRS, Université Paris-Saclay, Évry, France \\ ฯ Sondra, CentraleSupélec, Université Paris-Saclay, Gif-sur-Yvette, France
}

\begin{abstract}
A new lower bound is proposed in this article. Like Levenshtein bound, it relates to the maximum correlation value (autocorrelation and cross-correlation) a set of sequences can achieve. The novelty introduced here is that each sequence is associated with a mismatched filter. The proposed bound is inspired from Levenshtein's, holds for any set of unimodular sequences and can be applied in both aperiodic and periodic cases. It appears that the obtained expression does not deviate a lot from the (matched) Levenshtein, which indicates that the use of a mismatched filter will not guarantee much better sidelobe performance, as the number fo sequences is significant, contrary to the popular belief.
\end{abstract}

Index Terms-Aperiodic correlation lower bound, correlation, Levenshtein bound, mismatched filter, periodic correlation lower bound, Welch bound

\section{INTRODUCTION}

C ORRELATION plays a crucial role in order to determine the performance a system can achieve. For example, in the field of telecommunications, performance of a DS-CDMA system (Direct-Sequence Code-Division Multiple Access) is directly linked to the highest correlation value of each spreading sequence [1], called the Peak-to-Sidelobe Level Ratio (PSLR). In radar systems also, low PSLR codes are desired so that small targets remain detectable in the presence of stronger targets and clutter. It is thus relevant to design a set of sequences with the lowest cross-correlation and autocorrelation values for cooperative systems (e.g. statistical MIMO radars [2]), as it may reduce detection errors - a small PSLR improves the discrimination capacity between a weak target and a stronger one - and increase the average Signal-to-Noise Ratio (SNR).

Alongside extensive works to find sequences with the lowest PSLR (e.g. see [3], [4]), some studies have been conducted to define l ower b ounds o $n$ the P SLR - in o ther w ords, the performance that might be achieved at best - , according to some parameters: the number of sequences, their length, the assumptions made on the sequences, the type of correlation, etc. This paper deals with correlations of a set of sequences (both periodic and aperiodic cases are concerned), meaning that each correlation sequence from a pair of the set (autocorrelation and/or cross-correlation, for every delay) has to be handled. Notice that it is different from the optimal codebook topic [5], [6], in which only the correlation at the zero-delay is considered.

Welch was the first to establish a lower bound on correlation sidelobes for a set containing $M$ sequences of length $N$, under the identical energy assumption [7]. About 25 years later, a new lower bound was found by Levenshtein [8], [9] but only in the aperiodic case. At first, this bound was restricted to binary sequences, but it was later proved to hold for any sequence sets over the complex roots of unity [10] and even later for unimodular sequences [11]. This bound is tighter than the Welch one in most of the cases [9], [10], [12].

Levenshtein's bound is only valid for the aperiodic correlation, encountered in practice in radar systems by applying a matched filter at the reception side. The matched filter is known for maximising the Signal-to-Noise Ratio in Gaussian noise, but it may suffer from high sidelobes. Hence, in certain applications, for instance in the presence of multiple targets or in a strong clutter, it is interesting to replace this matched filter by another, identified as a mismatched filter, that may provide a better PSLR at the cost of some Loss-in-Processing Gain (LPG) [13]. Alongside numerous papers on waveform diversity [14], [15], [16], mismatched filters have gained an increasing interest in the recent years. Most notably it is of importance in joint radar and communication applications, in which specific waveforms are required to transmit information, and where the effort on the sidelobe level will thus be mainly turned back to the reception filter [17], [18], [19]. For that matter, it may seem interesting to determine a lower bound on the minimum PSLR that can be achieved using such a filter. To our knowledge, this question remains open.

This paper provides new results that enable to handle this question. It formulates a lower bound on correlation values - whether periodic or aperiodic — in the mismatched filter case. More precisely, a set of sequences is considered; each is associated with a mismatched filter (hence defining a second set). The correlation between a sequence and its counterpart is still refered as an "autocorrelation" by extension, while the others are cross-correlations. The main result of this paper is a lower bound on the maximum sidelobe value of all of these correlation sequences. Like Levenshtein's bound, it introduces some weights on the correlation sequences, so that an optimal weight vector may be calculated. The proof of this novelty is also inspired from Levenshtein's. But it holds for any set of 
unimodular sequences, with any restrictive constraints on the mismatched filter set.

Note that computation of both sets of sequences is not the concern of the paper. Nevertheless, there is a lot of literature on related topics, especially in the field of waveform diversity: the aforementioned search of pseudo-orthogonal sequences [20], [16], [21]; the generation of the optimal mismatched filter of a sequence [13] (optimal according to a criterion like the PSLR for instance) ; the joint optimisation of a sequence and its filter [22], [23], [24]; the definition of a s et of s equences and their associated mismatched filters [14], [15]. In practice, they help to get closer to their corresponding bounds but, akin to the matched filter $\mathrm{c}$ ase, these $\mathrm{c}$ annot probably be equalized.

This article is organised as follows. Section II provides a brief overview of some of the existing bounds in the matched filter case: the Welch bound and the Levenshtein bound. A new lower bound on the maximum correlation sidelobe with a set of mismatched filter is formulated in S ection III. S ection IV suggests some interpretations, especially by comparing the new result with existing bounds from the matched filter case. Some details on the proofs are given in the appendices.

Notation: In the following, vectors and matrices are designated with bold lowercase letters $(e . g . x)$ and bold uppercase letters (e.g. $\boldsymbol{X}$ ) respectively. Additionally, $x_{i}, \boldsymbol{X}_{i}$ and $X_{i, j}$ refer to the $i$-th element of vector $\boldsymbol{x}$, the $i$-th row and the element in the $i$-th row and $j$-th column of matrix $\boldsymbol{X}$, respectively. $(.)^{*},(.)^{T}$ and $(.)^{H}$ denote the conjugate, the transpose and the transpose conjugate operator, respectively.

Given an $m \times n$ matrix $\boldsymbol{X}$, its trace and its rank is denoted by $\operatorname{Tr}(\boldsymbol{X})$ and $\operatorname{rank}(\boldsymbol{X})$, whilst its Frobenius norm is $\|\boldsymbol{X}\|_{F}$. 。 stands for the Hadamard product (the entrywise product); by extension, the operator $(.)^{\circ q}$ refers to the Hadamard product of power $q$, i.e. $\boldsymbol{X}^{\circ q}=\boldsymbol{X} \circ \cdots \circ \boldsymbol{X}$ ( $q$ times). Circulant matrices are defined through a map denoted circ, a nd a re s pecified by a vector $\underset{\mathbb{C}^{n}}{\boldsymbol{x}}=\left[\underset{\mathbb{C}}{x_{1} \times \dot{n}}, x_{n}\right]^{T}$ of length $n$ :

$$
\boldsymbol{x} \mapsto \operatorname{circ}(\boldsymbol{x})=\left[\begin{array}{ccccc}
x_{1} & x_{2} & \ldots & x_{n-1} & x_{n} \\
x_{n} & x_{1} & x_{2} & & x_{n-1} \\
\vdots & x_{n} & x_{1} & \ddots & \vdots \\
x_{3} & & \ddots & \ddots & x_{2} \\
x_{2} & x_{3} & \ldots & x_{n} & x_{1}
\end{array}\right]
$$

$\lceil$.$\rceil denotes the ceil function. Finally, for two integers a$ and $b$, the modulo operator is denoted by $\bmod$, while $\llbracket \cdot, \cdot \rrbracket$ is an integer interval, that is, with $a<b, \llbracket a, b \rrbracket=\{a, a+1, \ldots, b\}$.

\section{REView on EXisting Bounds in ThE MAtcheD FILTER CASE}

\section{A. Definitions}

Let $\left\{\boldsymbol{x}^{m} \in \mathbb{C}^{N}\right\}_{m \in \llbracket 1, M \rrbracket}$ be a set of $M$ polyphase sequences of length $N$. Each sequence of the set is unimodular, meaning that any $n$-th element of any $m$-th sequence satisfies $\left|x_{n}^{m}\right|^{2}=1 / N$, for each $m \in \llbracket 1, M \rrbracket$ and $n \in \llbracket 0, N-1 \rrbracket$.
Therefore, their energy is constant:

$$
\sum_{n=0}^{N-1}\left|x_{n}^{m}\right|^{2}=1, \quad \forall m \in \llbracket 1, M \rrbracket .
$$

The correlation function may be expressed into two types, periodic and aperiodic, according to the definition of each sequence of $\left\{\boldsymbol{x}^{m}\right\}_{m}$ outside its original support. More precisely, for any $n<0$ or $n \geq N$ :

$$
x_{n}^{m}= \begin{cases}x_{(n \bmod N)}^{m} & \text { in the periodic correlation, } \\ 0 & \text { in the aperiodic correlation. }\end{cases}
$$

The correlation output between two sequences $\boldsymbol{x}^{m}$ and $\boldsymbol{x}^{l}$ is also a sequence, denoted $\boldsymbol{\theta}^{m, l}$. At delay $k$, it is defined by:

$$
\theta_{k}^{m, l}:=\sum_{n=0}^{N-1} x_{n-k}^{m}\left(x_{n}^{l}\right)^{*}, \text { for }|k|<N
$$

The latter is read as a cross-correlation if both sequences are different, while as an autocorrelation if $m=l$. Besides, it can be noticed that each autocorrelation mainlobe has the same value: $\theta_{0}^{m, m}=1$, for each $m \in \llbracket 1, M \rrbracket$.

\section{B. Lower Bounds on the PSLR}

Several lower bounds have been developed on the maximum sidelobe level of every auto- and cross-correlations. This level will be denoted in this paper by $\theta_{\max }$ and is provided by:

$$
\theta_{\max }^{2}=\max \left\{\max _{\substack{|k|<N \\ m_{1} \neq m_{2}}}\left|\theta_{k}^{m_{1}, m_{2}}\right|^{2}, \max _{\substack{k \neq 0 \\ m_{1}}}\left|\theta_{k}^{m_{1}, m_{1}}\right|^{2}\right\} .
$$

Notice that Eq. (4) includes every delay $k$, and not only the zero-delay (which is related to the inner product).

The Peak-to-Sidelobe Level Ratio, denoted by PSLR is defined as the ratio between the maximum sidelobe level and the peak level. Because of (1), it simply reduces to $\theta_{\max }^{2}$.

The most well-known bounds on the PSLR were provided by Welch [7] and Levenshtein [8] ${ }^{1}$. The former is expressed under the unimodular hypothesis by:

$$
\operatorname{PSLR} \geq \frac{M-1}{M L-1},
$$

with

$$
L= \begin{cases}N & \text { in the periodic case } \\ 2 N-1 & \text { in the aperiodic case }\end{cases}
$$

In 1999, Levenshtein has established another bound [8] that can be applied in the aperiodic case only. Originally designed for a set of binary sequences, it actually also holds for any unimodular polyphase sequences [11]. This bound is:

$$
\operatorname{PSLR} \geq \frac{1}{N^{2}}\left(N-\frac{Q_{2 N-1}\left(\boldsymbol{w}, \frac{N(N-1)}{M}\right)}{1-\sum_{i=1}^{2 N-1} w_{i}^{2} / M}\right)
$$

\footnotetext{
${ }^{1}$ In the literature, both bounds have been applied in the optimal codebook topic, see e.g. [5], [6]. It is not the concern of this paper because it only considers inner products, contrary to Eq. (4).
} 
where:

$$
\left\{\begin{aligned}
Q_{2 N-1}(\boldsymbol{w}, a) & =a \sum_{i=1}^{2 N-1} w_{i}^{2}+\sum_{s, t=1}^{2 N-1} l_{s, t, N} w_{s} w_{t}, \\
l_{s, t, N} & =\min (|s-t|, 2 N-1-|s-t|) .
\end{aligned}\right.
$$

$\boldsymbol{w}$ is a weight vector - on each correlation delay - that satisfies the following weighting condition:

$$
\left\{\begin{aligned}
\sum_{i=1}^{2 N-1} w_{i} & =1 \\
w_{i} \geq 0 & \text { for } i \in \llbracket 1,2 N-1 \rrbracket .
\end{aligned}\right.
$$

This bound has been proved to be tighter than the Welch bound in some cases ${ }^{2}$ : for $N \geq 2$ with $M=2, N \geq 3$ with $M=3$ and for $N \geq 2$ with $M \geq 4$. Some current literature [10], [12], [25] has been interested in the optimisation of this weight vector, in order to enhance the Levenshtein bound. Notably the authors of [25] have proposed an asymptotically locally optimal weight vector - in the particular case where each correlation delay is considered.

\section{Mismatched FiLTer Bound}

In this section is introduced a new bound on the PSLR for the output of the correlations between a set of polyphase sequences and a set of associated mismatched filters. This bound is defined in both periodic and aperiodic cases. Some proofs are given in the appendices.

\section{A. Definitions}

Akin to Section II-A, some definitions are given below.

1) A set of sequences: Let $\left\{\boldsymbol{x}^{m} \in \mathbb{C}^{N_{x}}\right\}_{m \in \llbracket 1, M \rrbracket}$ be a set of $M$ polyphase unimodular sequences of length $N_{x}$, with same energy equal to 1 .

2) A set of mismatched filters: Let $\left\{\boldsymbol{y}^{m} \in \mathbb{C}^{N_{y}}\right\}_{m \in \llbracket 1, M \rrbracket}$ be a set of mismatched filters. For simplicity, it will be assumed that the length $N_{y}$ of the mismatched filter sequences is equal to $N_{y}:=N_{x}+2 N_{s}, N_{s} \in \mathbb{N}$.

As implicitly mentioned in the introduction, a mismatched filter is a substitute for a matched filter, meaning that it should also help to detect a known signal (while rejecting the others). In other words, for each $m, l \in \llbracket 1, M \rrbracket, m \neq l$, the inner product $^{3}$ between a sequence $\boldsymbol{x}^{m} \ldots$

- ... and its so-called associated mismatched filter $\boldsymbol{y}^{m}$ should be maximised,

- ... and another filter $y^{l}$ should be minimised.

In practice, mismatched filters can be generated ${ }^{4}$ for example via a convex optimisation [13], [22], [14].

\footnotetext{
${ }^{2}$ Hence the Welch bound cannot be achieved in the aperiodic case. And, to the best of our knowledge, the Levenshtein bound has not been reached yet.

${ }^{3}$ Sequences $\boldsymbol{x}^{m}$ should of course be zero-padded if needed.

${ }^{4}$ While defining the bound, only the hypotheses in this section are needed: it is not required to exhibit the set of mismatched filters. On the other side, the bound calculation does not help to deduce the optimal set.
}

3) Correlations: Aperiodic and periodic correlations are defined according to the definition of each sequence $\boldsymbol{x}^{m}$, see (2). The output of the correlation between the $m$-th sequence $\boldsymbol{x}^{m}$ and the $l$-th mismatched filter $\boldsymbol{y}^{l}$ is also a sequence, denoted by $\boldsymbol{\theta}^{m, l} \in \mathbb{C}^{N_{x}+N_{y}-1}$ :

$$
\theta_{k}^{m, l}=\sum_{n=0}^{N_{y}-1} x_{n-k-N_{s}}^{m}\left(y_{n}^{l}\right)^{*}, \text { for }|k| \leq N_{x}+N_{s}-1 \text {. }
$$

Set $N_{k}:=N_{x}+N_{s}-1$.

4) Mainlobe, sidelobe: The mainlobe is simply defined as the central value of each correlation sequence $\boldsymbol{\theta}^{m, m}$ between a sequence $\boldsymbol{x}^{m}$ and its associated mismatched filter $\boldsymbol{y}^{m}, m \in$

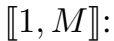

$$
\theta_{0}^{m, m}=\sum_{n=0}^{N_{y}-1} x_{n-N_{s}}^{m}\left(y_{n}^{m}\right)^{*}=\left|\theta_{0}^{m, m}\right| \mathrm{e}^{j \varphi_{m}},
$$

where $\varphi_{m} \in[-\pi, \pi]$ is the phase of $\theta_{0}^{m, m}$. Since phase shifting each mismatched filter $\boldsymbol{y}^{m}$ by $-\varphi_{m}$ will affect neither any sidelobe level nor the loss in processing gain, it is always possible to find a new set of mismatched filter $\left\{\boldsymbol{y}^{\prime m}\right\}_{m \in \llbracket 1, M \rrbracket}$ such that:

$$
\begin{aligned}
& \left\{\boldsymbol{y}^{\prime m}=\boldsymbol{y}^{m} \mathrm{e}^{-j \varphi_{m}}, m \in \llbracket 1, M \rrbracket\right\}, \\
& {\theta_{0}^{\prime m, m}}^{m, m}=\sum_{n=0}^{N_{y}-1} x_{n-N_{s}}^{m}\left(y_{n}^{\prime m}\right)^{*}=\left|\theta_{0}^{m, m}\right| \in \mathbb{R}^{+} .
\end{aligned}
$$

It is clear that $\boldsymbol{y}^{m}$ and $\boldsymbol{y}^{\prime m}$ present the same sidelobes (except for a constant phase). This property will be necessary in the following proof.

5) Peak-to-Sidelobe Level Ratio: Using the same notation as Section II-B, the Peak-to-Sidelobe Level Ratio (PSLR) is expressed by:

$$
\operatorname{PSLR}=\frac{\theta_{\max }^{2}}{\max _{m}\left(\left|\theta_{0}^{m, m}\right|^{2}\right)}
$$

where $\theta_{\max }^{2}$ is defined as in Eq. (4).

6) Loss-in-Processing-Gain (LPG): As already mentioned in the introduction, using a mismatched filter necessarily implies a loss in processing gain. This loss is defined by:

$$
\mathrm{LPG}_{m}=\frac{\left|\theta_{0}^{m, m}\right|^{2}}{E_{\boldsymbol{y}^{m}}^{2}}
$$

where $E_{\boldsymbol{y}^{m}}^{2}:=\left(\boldsymbol{y}^{m}\right)^{H} \boldsymbol{y}^{m}$ is the energy of $\boldsymbol{y}^{m}$. If each filter is designed as to provide a predefined mainlobe level $\theta_{0}^{m, m}$ (say equal to 1 ), then it clearly comes from its definition that the LPG is fully defined by the energy of the filter. In other words, an identical LPG for all these filters necessarily implies that each filter has the same energy. For now, no hypothesis has been set on each variable of Eq. (14). 
7) Mismatched filter l ength: In the periodic c ase, it is not relevant to consider mismatched filters that are larger than $N_{x}$. With the assumption that $y_{n}^{l}=0$ if $n \geq N_{y}$, Condition (2) indeed implies:

$$
\begin{aligned}
\theta_{k}^{m, l} & =\sum_{n=0}^{N_{y}-1} x_{n-k-N_{s}}^{m}\left(y_{n}^{l}\right)^{*} \\
& =\sum_{\kappa=0}^{\left\lceil N_{y} / N_{x}-1\right\rceil} \sum_{n=0}^{N_{x}-1} x_{n+\kappa N_{x}-k-N_{s}}^{m}\left(y_{n+\kappa N_{x}}^{l}\right)^{*} \\
& =\sum_{n=0}^{N_{x}-1} x_{n-k-N_{s}} \sum_{\kappa=0}^{\left\lceil N_{y} / N_{x}-1\right\rceil}\left(y_{n+\kappa N_{x}}^{l}\right)^{*} .
\end{aligned}
$$

Hence a mismatched filter $\boldsymbol{y}^{l}$ of length $N_{y}$ is equivalent to a mismatched filter $\boldsymbol{y}^{\prime}$ of length $N_{x}$, which coordinates are:

$$
y_{n}^{\prime}=\sum_{\kappa=0}^{\left\lceil N_{y} / N_{x}-1\right\rceil} y_{n+\kappa N_{x}}^{l} .
$$

\section{B. A New Bound on the PSLR}

A new lower bound is introduced now. It concerns the maximum correlation value of a set of unimodular sequences associated to a set of mismatched filters.

In the following are considered only sets of sequences $\left\{\boldsymbol{x}^{m}\right\}_{m \in \llbracket 1, M \rrbracket}$ and filters $\left\{\boldsymbol{y}^{m}\right\}_{m \in \llbracket 1, M \rrbracket}$ such that each mainlobe element $\theta_{0}^{m, m}$ is real positive. This hypothesis seems restrictive at first. But, as mentioned in Section III-A4, any sequence/filter pair may be resolved in that case by a simple phase shift (on the filter, for instance). This assertion is clearly stated in Proposition 1.

Proposition 1. (Extension of the bound to any complex filter) Let us assume that $\theta_{\max }^{2} \geq B$ for any mismatched filter set $\left\{\boldsymbol{y}^{\prime m}\right\}_{m}$ such that $\theta_{0}^{m, m}$ is real positive, for all $m \in \llbracket 1, M \rrbracket$. Then, the lower bound $B$ also applies for any other filter set $\left\{\boldsymbol{y}^{m}\right\}_{m}$ such that $\theta_{0}^{m, m}$ is complex.

Proof. Assume it exists a set of mismatched filters $\left\{\boldsymbol{y}^{m}\right\}_{m}$ such that its maximum sidelobe $\theta_{\max }^{2}$ satisfies $\theta_{\max }^{2}<B$. From each filter $\boldsymbol{y}^{m}, m \in \llbracket 1, M \rrbracket$, write:

$$
\theta_{0}^{m, m}:=\rho^{m} \mathrm{e}^{j \varphi_{m}} \quad \text { with } \rho^{m} \in \mathbb{R}^{+} \text {. }
$$

Then, let us consider another filter set $\left\{\boldsymbol{y}^{\prime m}\right\}_{m}$ such that each mainlobe is real positive, that is:

$$
\begin{aligned}
\boldsymbol{y}^{\prime m} & :=\boldsymbol{y}^{m} \mathrm{e}^{-j \varphi_{m}}, \\
\Longrightarrow \theta_{0}^{\prime m, m} & =\rho^{m} .
\end{aligned}
$$

It is clear that each filter $\boldsymbol{y}^{\prime m}$ shares the same sidelobe level as $\boldsymbol{y}^{m}$, so its maximum sidelobe should satisfy $\theta_{\max }^{2}<B$. However, since $\theta_{0}^{\prime m, m}$ is real positive, it should also satisfy ${\theta^{\prime}}_{\max }^{2} \geq B$ by hypothesis. Therefore, the initial claim is absurd: it cannot exist a set such that $\theta_{\max }^{2}<B$.

Let $\boldsymbol{X}^{m}$ and $\boldsymbol{Y}^{l}$ be two square matrices, $(m, l) \in \llbracket 1, M \rrbracket^{2}$. Each matrix contains shifted versions of respectively $\boldsymbol{x}^{m}$ and $\boldsymbol{y}^{l}$, but its definition depends on the considered periodicity of the signal. In the aperiodic case, $\boldsymbol{X}^{m}$ and $\boldsymbol{Y}^{l}$ are circulant square matrices of order $N_{X}:=N_{x}+N_{y}-1$ :

$$
\left\{\begin{aligned}
\boldsymbol{X}^{m} & =\operatorname{circ}\left(\left[\boldsymbol{x}^{m}, \mathbf{0}_{N_{y}-1,1}\right]\right) \\
& =\operatorname{circ}\left(\boldsymbol{X}_{1}^{m}\right), \\
\boldsymbol{Y}^{l} & =\operatorname{circ}\left(\boldsymbol{Y}_{1}^{l}\right),
\end{aligned}\right.
$$

where $\mathbf{0}_{m, n}$ denotes the zero matrix of size $m \times n$. In the periodic case, these matrices are of size $N_{X}:=N_{x}(c f$. III-A7):

$$
\left\{\begin{aligned}
\boldsymbol{X}^{m} & =\operatorname{circ}\left(\boldsymbol{x}^{m}\right), \\
\boldsymbol{Y}^{l} & =\operatorname{circ}\left(\boldsymbol{y}^{l}\right) .
\end{aligned}\right.
$$

For the sake of clarity, Eq. (19) is detailed below.

$\boldsymbol{X}^{m}=$

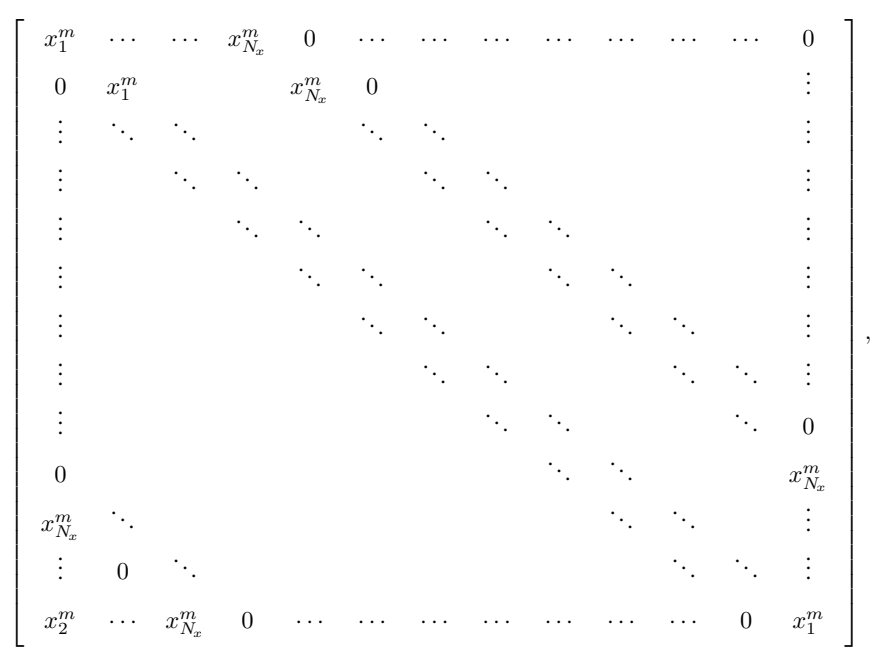

$\boldsymbol{Y}^{l}=$

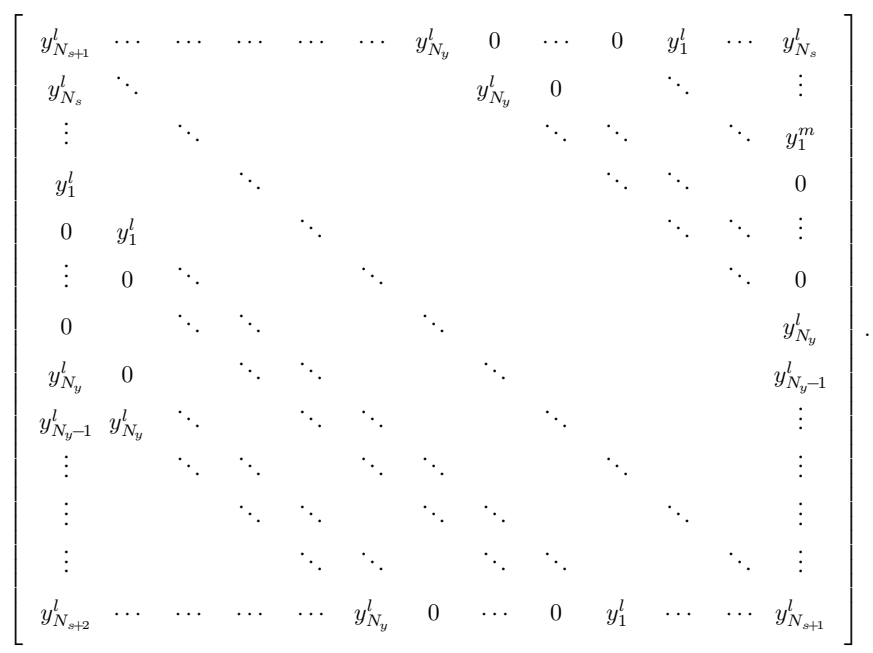

Consider the following matrices $\boldsymbol{X}$ and $\boldsymbol{Y}$ of size $M N_{x} \times N_{x}$ :

$$
\boldsymbol{X}=\left[\begin{array}{c}
\boldsymbol{X}^{1} \\
\boldsymbol{X}^{2} \\
\vdots \\
\boldsymbol{X}^{M}
\end{array}\right], \quad \boldsymbol{Y}=\left[\begin{array}{c}
\boldsymbol{Y}^{1} \\
\boldsymbol{Y}^{2} \\
\vdots \\
\boldsymbol{Y}^{M}
\end{array}\right]
$$

All the correlation values are contained in a square matrix $\boldsymbol{R}$ of order $M N_{x}$. 
It is defined by:

$$
\boldsymbol{R}=\boldsymbol{X} \boldsymbol{Y}^{H}=\left[\begin{array}{ccc}
\boldsymbol{X}^{1}\left(\boldsymbol{Y}^{1}\right)^{H} & \cdots & \boldsymbol{X}^{1}\left(\boldsymbol{Y}^{M}\right)^{H} \\
\vdots & \ddots & \vdots \\
\boldsymbol{X}^{M}\left(\boldsymbol{Y}^{1}\right)^{H} & \cdots & \boldsymbol{X}^{M}\left(\boldsymbol{Y}^{M}\right)^{H}
\end{array}\right]
$$

such that:

$$
\boldsymbol{X}^{m}\left(\boldsymbol{Y}^{l}\right)^{H}=\operatorname{circ}\left(\left[\theta_{0}^{m, l}, \theta_{-1}^{m, l}, \ldots, \theta_{-N_{k}}^{m, l}, \theta_{N_{k}}^{m, l}, \ldots, \theta_{1}^{m, l}\right]\right) .
$$

It is also possible to weight each correlation value, akin to Levenshtein bound (7). Each row of $\boldsymbol{X}^{m}$ and $\boldsymbol{Y}^{l}$ is weighted by a coefficient $w_{i}$, thus becoming:

$$
\left\{\begin{aligned}
\tilde{\boldsymbol{X}}_{i}^{m} & :=\boldsymbol{X}_{i}^{m} \sqrt{w_{i}}, \\
\tilde{\boldsymbol{Y}}_{i}^{l} & :=\boldsymbol{Y}_{i}^{l} \sqrt{w_{i}} \text { for } i \in \llbracket 1, N_{X} \rrbracket,(l, m) \in \llbracket 1, M \rrbracket^{2},
\end{aligned}\right.
$$

where the weight vector $\boldsymbol{w}:=\left\{w_{i}\right\}_{\tilde{c}=1}^{N_{x}}$ satisfies the weighting condition (9). Matrices $\tilde{\boldsymbol{X}}, \tilde{\boldsymbol{Y}}$ and $\tilde{\boldsymbol{R}}$ are constructed as above:

$$
\tilde{\boldsymbol{R}}=\tilde{\boldsymbol{X}} \tilde{\boldsymbol{Y}}^{H} \text { with } \tilde{\boldsymbol{X}}=\left[\begin{array}{c}
\tilde{\boldsymbol{X}}^{1} \\
\vdots \\
\tilde{\boldsymbol{X}}^{M}
\end{array}\right] \text { and } \tilde{\boldsymbol{Y}}=\left[\begin{array}{c}
\tilde{\boldsymbol{Y}}^{1} \\
\vdots \\
\tilde{\boldsymbol{Y}}^{M}
\end{array}\right] \text {, }
$$

such that $\tilde{\boldsymbol{R}}$ contains now a weighted version of all correlation values. Remark that it is quite common to consider weights that only handle the the $K$-first delays:

$$
\sum_{i=1}^{K} w_{i}=1 \text { such that } \begin{cases}w_{i} \geq 0 & i \in \llbracket 1, K \rrbracket, \\ w_{i}=0 & i>K \text { or } i<0 .\end{cases}
$$

Under these hypotheses, an upper and a lower bound on the Frobenius norm of $\tilde{\boldsymbol{R}},\|\tilde{\boldsymbol{R}}\|_{F}^{2}$, is suggested in the following.

Lemma 1. (Upper Bound) Under the above-mentioned hypotheses, an upper bound of the Frobenius norm of matrix $\tilde{\boldsymbol{R}}$ is the following:

$$
\|\tilde{\boldsymbol{R}}\|_{F}^{2} \leq \sum_{m=1}^{M}\left(\left|\theta_{0}^{m, m}\right|^{2}-\theta_{\max }^{2}\right) \boldsymbol{w}^{T} \boldsymbol{w}+M^{2} \theta_{\max }^{2} .
$$

Proof. This result is similar to [9, Lemma 1] and is obtained by a similar proof.

In addition, a lower bound on the Frobenius norm of matrix $\tilde{\boldsymbol{R}}$ can also be computed.

Lemma 2. (Lower bound) Under the above-mentioned hypotheses, a lower bound of the Frobenius norm of the matrix $\tilde{\boldsymbol{R}}$ is the following:

$$
\|\tilde{\boldsymbol{R}}\|_{F}^{2} \geq \frac{\left|\sum_{m=1}^{M} \theta_{0}^{m, m}\right|^{2}}{l_{N_{x}, N_{y}, K}}
$$

with $l_{N_{x}, N_{y}, K}=\min \left(N_{x}+N_{y}-1, N_{x}+K-1, M K\right)$.

Proof. See Appendix A.

By gathering these lemmas, a lower bound of the maximum correlation sidelobe $\theta_{\max }$ can easily be deduced. Its expression is given in the following theorem:

Theorem 1. For any set of $M$ unimodular sequences of length $N_{x}$ under the identical energy assumption, for any set of associated mismatched filter of length $N_{y}$, a lower bound of the maximum correlation sidelobe $\theta_{\max }$ is:

$$
\begin{aligned}
\theta_{\max }^{2} \geq & \frac{1}{M^{2}-M \boldsymbol{w}^{T} \boldsymbol{w}} \\
& \times\left[\frac{\left|\sum_{m=1}^{M} \theta_{0}^{m, m}\right|^{2}}{l_{N_{x}, N_{y}, K}}-\boldsymbol{w}^{T} \boldsymbol{w} \sum_{m=1}^{M}\left|\theta_{0}^{m, m}\right|^{2}\right]
\end{aligned}
$$

with: $\boldsymbol{w}$ a weight vector of length $N_{X}$ that satisfies (9),

$$
\begin{aligned}
& l_{N_{x}, N_{y}, K}=\min \left(N_{x}+N_{y}-1, N_{x}+K-1, M K\right), \\
& K \in \llbracket 1, N_{X} \rrbracket .
\end{aligned}
$$

Theorem 1 establishes a bound for a given $K$. Actually, a tighter bound can be obtained, considering those with a smaller $K$, that is:

$$
\begin{aligned}
\theta_{\max }^{2} \geq \max _{\substack{K^{\prime} \\
K^{\prime} \leq K}} \frac{1}{M^{2}-M \boldsymbol{w}^{T} \boldsymbol{w}} \\
\quad \times\left[\frac{\left|\sum_{m=1}^{M} \theta_{0}^{m, m}\right|^{2}}{l_{N_{x}, N_{y}, K^{\prime}}}-\boldsymbol{w}^{T} \boldsymbol{w} \sum_{m=1}^{M}\left|\theta_{0}^{m, m}\right|^{2}\right] .
\end{aligned}
$$

Some papers may study a more general case, in which $\theta_{\text {max }}^{2 q}$ is lower-bounded instead, with $q \in \mathbb{N}^{*}$ (e.g. [26] with matched filters). The suggested bound can easily be extended to that case, and becomes:

Theorem 2. (General case, under the same hypotheses)

$$
\begin{aligned}
\theta_{\max }^{2 q} \geq & \frac{1}{M^{2}-M \boldsymbol{w}^{T} \boldsymbol{w}} \\
& \times\left[\frac{\left|\sum_{m=1}^{M} \theta_{0}^{m, m}\right|^{2}}{l_{N_{x}, N_{y}, K, q}}-\boldsymbol{w}^{T} \boldsymbol{w} \sum_{m=1}^{M}\left|\theta_{0}^{m, m}\right|^{2 q}\right]
\end{aligned}
$$

with: $\boldsymbol{w}$ a weight vector of length $N_{X}$ that satisfies (9),

$$
\begin{aligned}
& \text { a different weighting, since } \tilde{\boldsymbol{X}}_{i}^{m}=\boldsymbol{X}_{i}^{m} w_{i}^{1 / 2 q}, \\
& l_{N_{x}, N_{y}, K, q}=\min \left(\left(\begin{array}{c}
N_{X}+q-1 \\
q
\end{array}\right),\left(\begin{array}{c}
N_{x}+K+q-2 \\
q
\end{array}\right), M K\right), \\
& K \in \llbracket 1, N_{X} \rrbracket .
\end{aligned}
$$

Proof. See Appendix B.

\section{DisCUSSION AND INTERPRETATION}

Each bound introduced in the previous section depends on several parameters such as the number of sequences $M$, their length $N_{x}$, or the weight vector $\boldsymbol{w}$. In this section, a discussion is made on the influence of all these parameters. In particular, the existence of an optimal weight vector is explained. Some comparisons - especially with bounds on the matched filter — are made at the end.

\section{A. Optimal Weight Vector}

Consider the right-hand side of Eq. (30) of Theorem 1 as a function of the weight vector $\boldsymbol{w}$. This parameter is said to be "optimal" if it provides the tightest bound, i.e. the largest in value. 
Notice that (30) can be rewritten as:

$$
\begin{aligned}
\theta_{\max }^{2} \geq & \left(\frac{1}{M} \sum_{m=1}^{M}\left|\theta_{0}^{m, m}\right|^{2}\right) \times \\
& {\left[1-\frac{M}{M-\boldsymbol{w}^{T} \boldsymbol{w}} \frac{l_{N_{x}, N_{y}, K}-\frac{\left|\sum_{m=1}^{M} \theta_{0}^{m, m}\right|^{2}}{M \sum_{m=1}^{M}\left|\theta_{0}^{m, m}\right|^{2}}}{l_{N_{x}, N_{y}, K}}\right] . }
\end{aligned}
$$

It is now easier to observe that the lower $\boldsymbol{w}^{T} \boldsymbol{w}$, the higher the bound. This assertion is not necessarily true with Levenshtein bound, since $l_{s, t, N}$ (similar to $l_{N_{x}, N_{y}, K}$ ) depends on the weight vector. Optimizing $\boldsymbol{w}$ such that it minimizes $\boldsymbol{w}^{T} \boldsymbol{w}$ should thus give a tighter bound. The Cauchy-Schwarz inequality writes:

$$
\boldsymbol{w}^{T} \boldsymbol{w}=\sum_{i=1}^{K} w_{i}^{2} \geq \frac{\left(\sum_{i=1}^{K} w_{i}\right)^{2}}{K}=\frac{1}{K} .
$$

The best weight vector is obtained when the Cauchy-Schwarz inequality meets, that is to say when $\boldsymbol{w}^{T} \boldsymbol{w}=1 / K$. It is quite clear that the constant weight vector, recalled below, fill this requirement:

$$
w_{i}= \begin{cases}1 / K & \text { if } i \leq K \\ 0 & \text { otherwise }\end{cases}
$$

Using this particular weight vector, Theorem 1 becomes:

Corollary 1. (Maximum correlation sidelobe bound, constant weight vector)

$$
\begin{aligned}
\theta_{\max }^{2} \geq & \left(\frac{1}{M} \sum_{m=1}^{M}\left|\theta_{0}^{m, m}\right|^{2}\right) \times \\
& {\left[1-\frac{M K}{M K-1} \frac{l_{N_{x}, N_{y}, K}-\frac{\left|\sum_{m=1}^{M} \theta_{0}^{m, m}\right|^{2}}{M \sum_{m=1}^{M}\left|\theta_{0}^{m, m}\right|^{2}}}{l_{N_{x}, N_{y}, K}}\right] . }
\end{aligned}
$$

This result is really interesting. It means that there exists an optimal weight vector $\boldsymbol{w}$, contrary to the Levenshtein bound, in which the optimal weight vector is still a subject of research. (However, note that the authors of [25] have proposed a locally optimal weight vector, in the particular case $K=2 N-1$.)

In the previous expression, $\theta_{\max }^{2}$ is only determined by each mainlobe value $\theta_{0}^{m, m}$. Since $\theta_{0}^{m, m}$ is only expressed through the energy of the filters and the loss in processing gain that have not been constrained - it can be set to $\theta_{0}^{m, m}=1$ without loss of generality. Hence, the lower bound becomes:

$$
\theta_{\max }^{2} \geq \frac{M K-l_{N_{x}, N_{y}, K}}{(M K-1) l_{N_{x}, N_{y}, K}} .
$$

\section{B. Bounds Comparisons}

In this section, the behaviour of the proposed bound is described according to some parameters it depends on. Besides, it will be compared with the matched filter bounds from Welch [7] and Levenshtein [9].
1) Bound value: Like the Levenshtein bound, the proposed one - Eq. (37) - is defined with a parameter $K$, the number of considered correlation values. Fig. 1 depicts its effect in a simple example: a set of $M=4$ sequences of length $N_{x}=$ 1024. In this figure, it seems that the length of the mismatched filter $N_{y}$ has an influence on the choice of $K$. Its optimal value is indeed determined as the one that gives the tightest bound, in other words, the greatest value in each curve. In the case $N_{y}=$ $3 N_{x}$, the optimal value is not achieved for $K=K_{\max }:=$ $N_{x}+N_{y}-1$, meaning that considering every correlation value is not necessarily useful. Additionally, as expected, a longer filter goes with a looser bound (a noticeable difference of 1.15 $\mathrm{dB}$ here). Does this observation always hold? Check another example, still with $N_{x}=1024$, but with many more sequences $(M=10)$.

Both curves of Fig. 2 show identical bounds, despite different mismatched filter lengths. In this example, each bound appears to be independent of $N_{y}$, meaning that a filter larger than $N_{x}$ does not provide any enhancement. This phenomenon has already been seen in the periodic case (see Section III-A7) and may be explained here via $l_{N_{x}, N_{y}, K}$ (cf. Lemma 2). As illustrated in Fig. 3 (left) with a plain line, this parameter is in a way piecewise linear as a function of $K$. But the bound reaches its maximum at $K_{\mathrm{opt}}<N_{y}$, while the corresponding "piece" does not depend on $N_{y}$. Anyway, notice here an improvement of $1.3 \mathrm{~dB}$ compared to the matched filter bound.

2) Comparison with matched filter bounds: Even though their proofs are quite similar, Theorem 1 does not "include" the earlier matched bounds (e.g. Levenshtein [9]) per se. That can easily be seen with Figs. 1 and 2: neither their respective curves are overlaid nor their highest values (i.e. the bound value) are equal.

However, the matched filter is after all a particular mismatched filter. So, theoretically, Theorem 1 could be employed under its constraints, that are ${ }^{5}$ :

1) Same length: $N_{x}=N_{y}$;

2) Zero loss: $\left|\theta_{0}^{m, m}\right|^{2}=1, \mathrm{LPG}_{m}=0$, for all $m \in \llbracket 1, M \rrbracket$. Actually, this is represented on the left of Figs. 1 and 2. But, as one could expect, Theorem $1-$ in this particular case is looser ${ }^{6}$. This difference can be explained with the structure of correlation sequences, which manifests in the proof of the lower bound, $c f$. Appendix A.

- In the matched filter case, there is only one set of unimodular sequences, $\left\{\boldsymbol{x}^{m} \in \mathbb{C}^{N_{x}}\right\}_{m \in \llbracket 1, M \rrbracket}$. Sidelobes that are evaluated in those bounds come from correlations between two sequences within the set. In addition, an autocorrelation sequence has some structural properties that are exploited in the proof ( $c f$. the lower bound of [11] and Lemma 2).

- In the mismatched filter case, there are two sets of sequences: $\left\{\boldsymbol{x}^{m} \in \mathbb{C}^{N_{x}}\right\}_{m \in \llbracket 1, M \rrbracket}$, composed of unimodular sequences, and $\left\{\boldsymbol{y}^{m} \in \mathbb{C}^{N_{y}}\right\}_{m \in \llbracket 1, M \rrbracket}$, in which no hypothesis is put on. Correlations between two sequences

\footnotetext{
${ }^{5}$ There is no way to easily incorporate the restrictive constraint $\boldsymbol{y}^{m}=\boldsymbol{x}^{m}$ (for all $m \in \llbracket 1, M \rrbracket$ ).

${ }^{6}$ It also means that a mismatched filter with the same length may provide some gain, compared to a matched filter.
} 

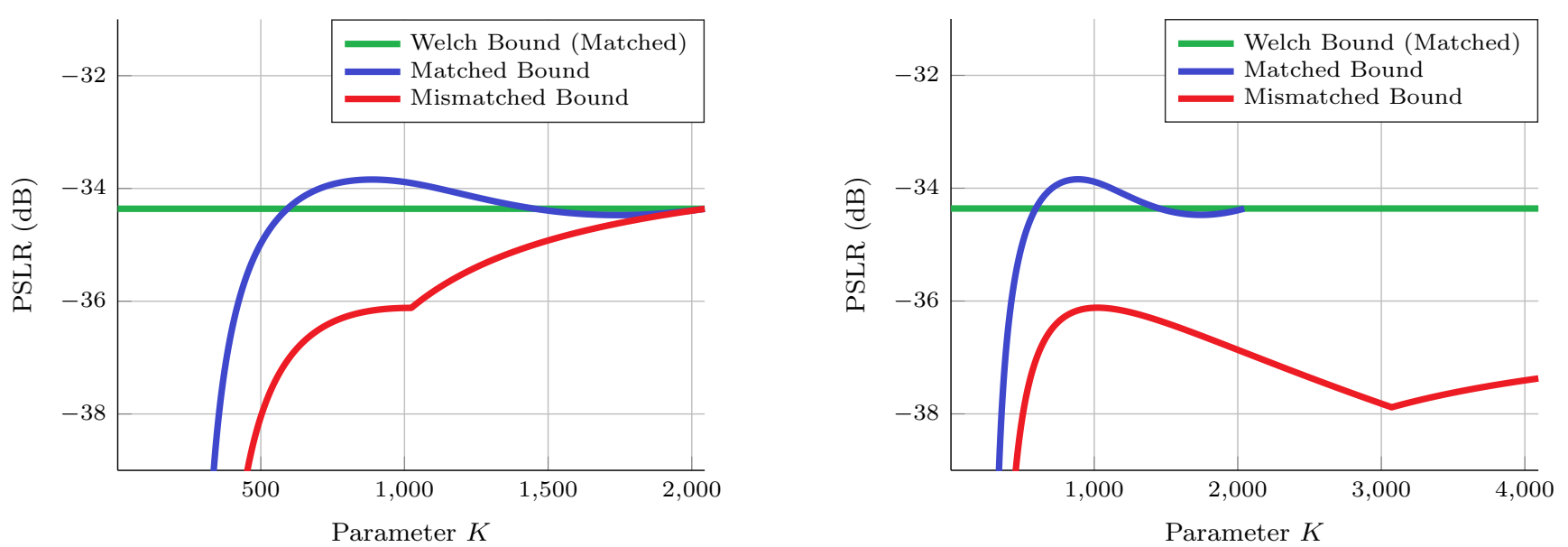

Fig. 1. Comparison of aperiodic correlation bounds, as functions of $K$, for a set of $M=4$ sequences. On the left: $N_{y}=N_{x}$. On the right: $N_{y}=3 N_{x}$.

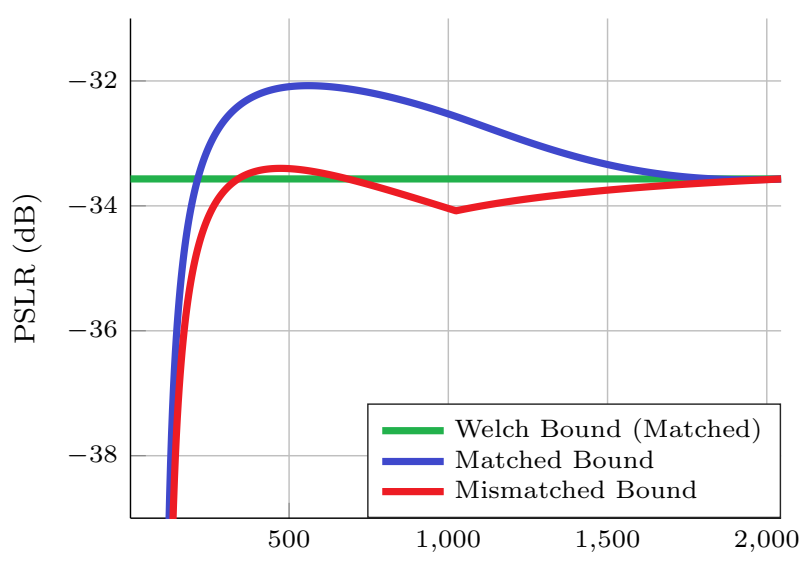

Parameter $K$

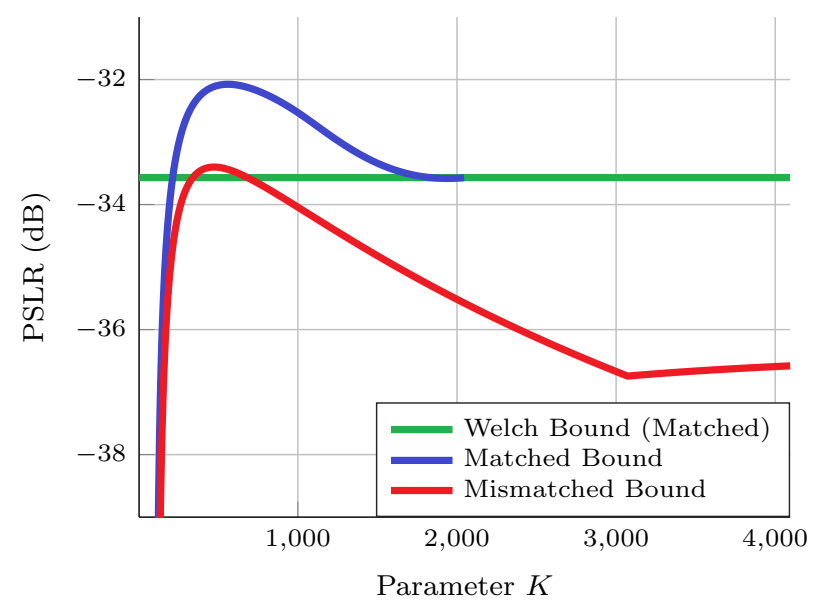

Fig. 2. Comparison of aperiodic correlation bounds as functions of $K$, for a set of $M=10$ sequences. On the left: $N_{y}=N_{x}$. On the right: $N_{y}=3 N_{x}$.
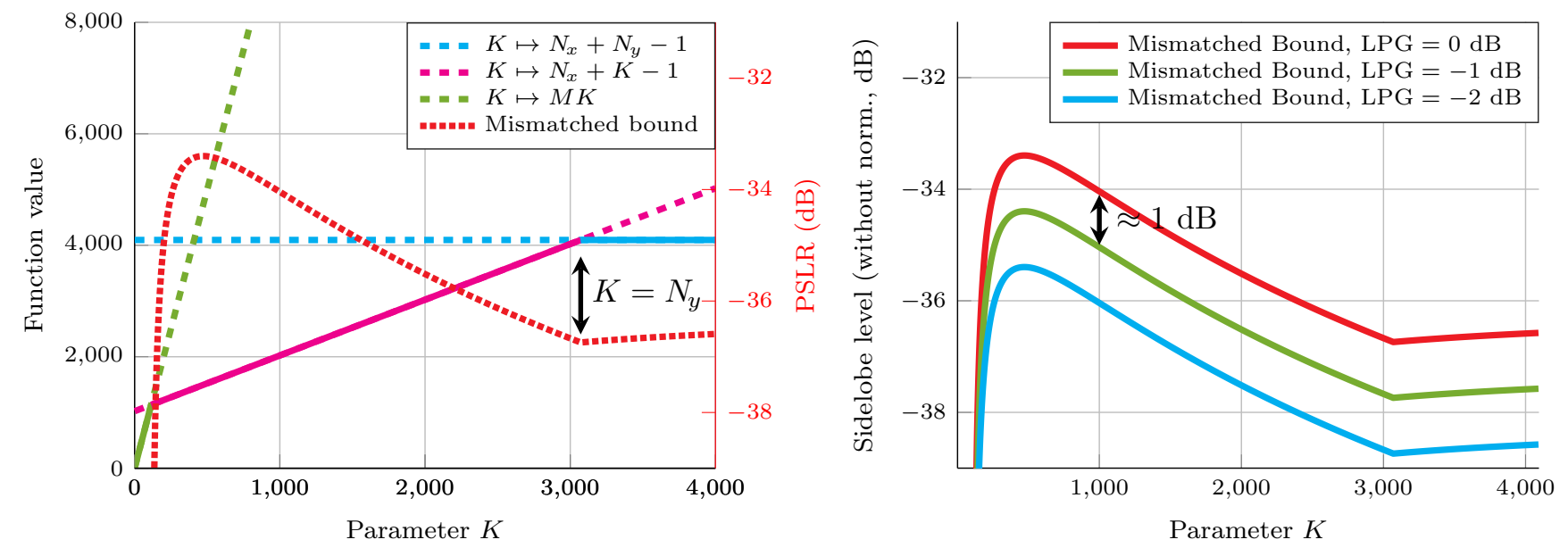

Fig. 3. Some annotations on Fig. 2 right, but with different scales. On the left: representation of each component of $l_{N_{x}, N_{y}, K}$. The latter, minimum of these three components, is drawn with a plain line. On the right: proposed bound with several LPGs and so several mainlobe levels. 


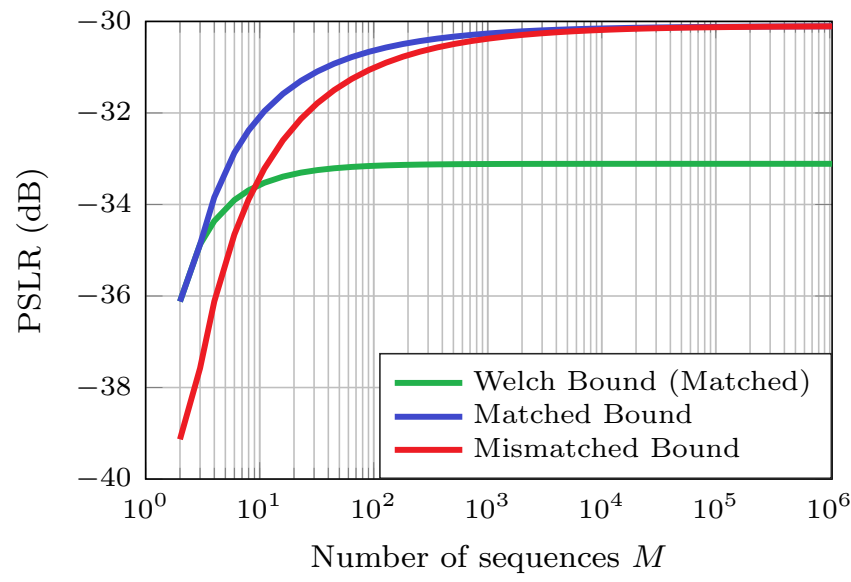

Fig. 4. Aperiodic correlation bound value $v s$. number of sequences $M$. Sequences of length $N_{x}=1024$. Mismatched filters three times longer.

of the same set are ignored. Instead, the mismatched filter bound studies the correlations between $\boldsymbol{x}^{m}$ and $\boldsymbol{y}^{l}$, for each $m, l \in \llbracket 1, M \rrbracket$. However, no useful property is known this time, to the best of our knowledge.

On the other side, it is confirmed that the Welch bound is too loose. Its expected performance must be indeed "worse" or, at best, equal. Nonetheless, Fig. 2 shows the opposite: the Welch bound is there smaller.

Finally, note that the Welch bound can be retrieved from the matched filter bound Eq. (7) or the mismatched filter one, in a peculiar case. Indeed, adding the following hypothesis that corresponds to Welch bound computation:

3) All correlation delays: $K=2 N_{x}-1$ (and again, with a constant weight vector),

it induces:

$$
\begin{aligned}
l_{N_{x}, N_{y}, K} & =\min \left(N_{x}+N_{y}-1, N_{x}+K-1, M K\right) \\
& =2 N_{x}-1 ;
\end{aligned}
$$

so that Eq. (37) writes:

$$
\begin{aligned}
\theta_{\max }^{2} & \geq \frac{M K-l_{N_{x}, N_{y}, K}}{(M K-1) l_{N_{x}, N_{y}, K}} \\
& =\frac{M-1}{M\left(2 N_{x}-1\right)-1},
\end{aligned}
$$

which is precisely the Welch bound, $c f$. Eq. (5).

3) Asymptotic behaviour: As expected, mismatched filters may provide some improvements, in terms of sidelobes. But, does this depend on the number of sequences of the set ? Let us find out with Fig. 4.

This figure demonstrates a not-so-surprising behaviour: the gain brought by mismatched filters compared to matched filters becomes smaller and smaller as the number of sequences increases. For small sets, mismatched filtering is fully realized (a difference of $3 \mathrm{~dB}$ for a set of 2 sequences of length $\left.N_{x}=1024\right)$ while for larger sets, it loses its appeal as both bounds are nearly equal. The cardinality of the set $M$ seems involved: although a mismatched filter provides some additional degrees of freedom (it is not concerned by the constant modulus constraint and its length $N_{y}$ may be greater than $N_{x}$ ), the number of constraints on the correlation sequences ( $M^{2}$ autocorrelation and cross-correlation sequences) grows quicker. Hence, the impact of this provided relaxation is poor and becomes insignificant, compared to the correlation constraints induced by the system.

4) Effects of the LPG: Recall that the loss-in-processing gain - LPG, Eq. (14) - is defined with the mainlobe level $\left|\theta_{0}^{m, m}\right|^{2}$ and the energy of each filter $E_{\boldsymbol{y}^{m}}^{2}$. In the proof, no hypothesis is put on all these elements. Both statements are thus equivalent:

(i) Set the mainlobe level $\theta_{0}^{m, m}$, and let both parameters $\mathrm{LPG}_{m}$ and $E_{\boldsymbol{y}^{m}}$ be undefined.

(ii) Normalise each mismatched filter, i.e. $E_{\boldsymbol{y}^{m}}^{2}=1$, which implies $\left|\theta_{0}^{m, m}\right|^{2}=\mathrm{LPG}_{m}$.

Figs. 1 and 2 considers $(i)^{7}$ with $\theta_{0}^{m, m}=1$, which yields to Eq. 37. In order to study the behaviour of the LPG, consider case $(i i)$. Let us set for simplicity, for all $m \in \llbracket 1, M \rrbracket$ :

$$
\left\{\begin{aligned}
E_{\boldsymbol{y}^{m}}^{2} & =1 & & \text { (filter normalisation) } \\
\alpha: & =\mathrm{LPG}_{m} & & \text { (identical loss) }
\end{aligned}\right.
$$

which implies $\left|\theta_{0}^{m, m}\right|^{2}=\alpha$. Hence, Corollary 1 becomes:

$$
\theta_{\max }^{2} \geq \alpha \frac{M K-l_{N_{x}, N_{y}, K}}{(M K-1) l_{N_{x}, N_{y}, K}},
$$

which is nearly Eq. (37), up to a factor $\alpha$. This indicates that LPGs only induce a shift of the bound.

In order to see that, Fig. 3 (right) represents Eq. (41) with different values of $\alpha$. Green curve is with zero loss, which is equivalent to the "original" bound. Notice the difference of around $1 \mathrm{~dB}$ with the blue curve $(\mathrm{LPG}=-1 \mathrm{~dB})$. However, in this case, the mainlobe value is also equal to $-1 \mathrm{~dB}$, because of Eq. (40). At the end of the day, the Peak-to-Sidelobe Level Ratio (PSLR) is the same... Finally, if the loss is taken into account, each curve is equivalent, which means:

- The proposed bound is true whatever the loss considered, provided that the constraint $\theta_{0}^{m, m}=1$ is satisfied (which does not imply that there is no loss, but which implies that the mismatched filter output for the zero delay must provide a mainlobe).

- Allowing a significant loss will not enable to lower the bound.

- This bound - the first on the mismatched filter case, to our knowledge - has no dependence with the LPG. Maybe another tighter bound will, but it is yet to be found.

All these comments concern how these bounds behave, but there is any mention on how loose they are. Previous figures have noticed that a mismatched filter that is longer than the initial sequence does not necessarily lead to a larger bound. However in practice, the additional degrees of freedom it procures may enable to get closer to these bounds, or at least may provide some gain. But probably quite poor...

\footnotetext{
${ }^{7}$ As the filter energy has not been set, this hypothesis does not mean that there is zero loss.
} 


\section{CONCLUSiON}

A new lower bound has been introduced in this paper. It estimates the maximum correlation value of a set of sequences with a set of associated mismatched filters. I ts expression is fairly general, as it holds for any unimodular sequences, it can be applied in both aperiodic and periodic cases, and there is no constraint on the mismatched filters.

The obtained result can be interpreted in several ways.

- On one hand, this bound is somehow an extension of Levenshtein's work to the more general case of unimodular sequences with corresponding mismatched filters. It enables to set a lower bound on the minimum sidelobe level that can be reached using such mismatched filters, and in that sense provides important indications when designing a given radar or joint communication and radar system. Interestingly, it appears that this mismatched bound does not deviate a lot from the matched Levenshtein, which indicates that the use of a mismatched filter will not guarantee much better sidelobe performance. While we have been able to link the proposed bound with the Welch bound, it has not been possible yet to provide a similar link with the Levenshtein bound. Finding such a generic bound that links both matched and mismatched filter may be of interest for some future work.

- On the other hand, each bound has still not be reached - to our knowledge - or can be possibly loose (take a look at their asymptotic behavior). Does that change with a mismatched filter?

\section{APPENDIX A}

PROOF OF LEMMA 2 (LOWER BOUND)

This appendix proves Property 2, recalled below :

Lemma 2. (Lower bound) Under the above-mentioned hypotheses, a lower bound of the Frobenius norm of the matrix $\tilde{\boldsymbol{R}}$ is the following:

$$
\|\tilde{\boldsymbol{R}}\|_{F}^{2} \geq \frac{\left|\sum_{m=1}^{M} \theta_{0}^{m, m}\right|^{2}}{l_{N_{x}, N_{y}, K}}
$$

with $l_{N_{x}, N_{y}, K}=\min \left(N_{x}+N_{y}-1, N_{x}+K-1, M K\right)$.

Proof. Let $\left\{\lambda_{i}, i \in \llbracket 1, \operatorname{rank}(\tilde{\boldsymbol{R}}) \rrbracket\right\}$ be the non-zero eigenvalues of $\tilde{\boldsymbol{R}}$. They may define the Frobenius norm of matrix $\tilde{\boldsymbol{R}}$ :

$$
\|\tilde{\boldsymbol{R}}\|_{F}^{2}=\sum_{i=1}^{\operatorname{rank}(\tilde{\boldsymbol{R}})}\left|\lambda_{i}\right|^{2}
$$

and its trace :

$$
\operatorname{Tr}(\tilde{\boldsymbol{R}})=\sum_{i=1}^{\operatorname{rank}(\tilde{\boldsymbol{R}})} \lambda_{i}
$$

that is also equal to, by construction :

$$
\begin{aligned}
\operatorname{Tr}(\tilde{\boldsymbol{R}}) & =\sum_{m=1}^{M} \sum_{i=1}^{N_{x}} \theta_{0}^{m, m} w_{i} \\
& =\sum_{m=1}^{M} \theta_{0}^{m, m} .
\end{aligned}
$$

The Cauchy-Schwarz inequality links both :

$$
\begin{aligned}
|\operatorname{Tr}(\tilde{\boldsymbol{R}})|^{2} & \leq \operatorname{rank}(\tilde{\boldsymbol{R}})\left(\sum_{i=1}^{\operatorname{rank}(\tilde{\boldsymbol{R}})}\left|\lambda_{i}\right|^{2}\right) \\
& \leq \operatorname{rank}(\tilde{\boldsymbol{R}})\|\tilde{\boldsymbol{R}}\|_{F}^{2} .
\end{aligned}
$$

Hence,

$$
\|\tilde{\boldsymbol{R}}\|_{F}^{2} \geq \frac{\operatorname{Tr}(\tilde{\boldsymbol{R}})^{2}}{\operatorname{rank}(\tilde{\boldsymbol{R}})}
$$

From there, it remains to find an upper bound of $\operatorname{rank}(\tilde{\boldsymbol{R}})$. Remind that $\tilde{\boldsymbol{R}}=\tilde{\boldsymbol{X}} \tilde{\boldsymbol{Y}}^{H}$. The particular structure of both matrices gives us (with $N_{y} \geq N_{x}$ ):

$$
\begin{aligned}
\operatorname{rank}(\tilde{\boldsymbol{X}}) & = \begin{cases}\min \left(M K, N_{x}+K-1\right) & \text { if } K \leq N_{y} \\
\min \left(M K, N_{x}+N_{y}-1\right) & \text { otherwise, }\end{cases} \\
\operatorname{rank}(\tilde{\boldsymbol{Y}}) & =\min \left(M K, N_{y}+\min \left(K, N_{x}\right)-1\right), \\
\operatorname{rank}(\tilde{\boldsymbol{R}}) \leq & \min (\operatorname{rank}(\tilde{\boldsymbol{X}}), \operatorname{rank}(\tilde{\boldsymbol{Y}})) \\
& =\min \left(M K, N_{x}+K-1, N_{x}+N_{y}-1\right) .
\end{aligned}
$$

Set $l_{N_{x}, N_{y}, K}:=\min \left(M K, N_{x}+K-1, N_{x}+N_{y}-1\right)$. Gathering (46) and (47) gives the desired result:

$$
\|\tilde{\boldsymbol{R}}\|_{F}^{2} \geq \frac{\left|\sum_{m=1}^{M} \theta_{0}^{m, m}\right|^{2}}{l_{N_{x}, N_{y}, K}}
$$

\section{APPENDIX B}

\section{Proof of TheOrem 2 (GENERAL CASE)}

This appendix deals with a generalisation of Theorem 1, where the term $\theta_{\max }^{2 q}$ has to be lower-bounded, $q \in \mathbb{N}^{*}$.

Theorem 2. (General case, under the same hypotheses)

$$
\begin{aligned}
\theta_{\max }^{2 q} \geq & \frac{1}{M^{2}-M \boldsymbol{w}^{T} \boldsymbol{w}} \\
& \times\left[\frac{\left|\sum_{m=1}^{M} \theta_{0}^{m, m}\right|^{2}}{l_{N_{x}, N_{y}, K, q}}-\boldsymbol{w}^{T} \boldsymbol{w} \sum_{m=1}^{M}\left|\theta_{0}^{m, m}\right|^{2 q}\right]
\end{aligned}
$$

with: $\boldsymbol{w}$ a weight vector of length $N_{X}$ that satisfies (9), a different weighting, since $\tilde{\boldsymbol{X}}_{i}^{m}=\boldsymbol{X}_{i}^{m} w_{i}^{1 / 2 q}$, $l_{N_{x}, N_{y}, K, q}=\min \left(\left(\begin{array}{c}N_{X}+q-1 \\ q\end{array}\right),\left(\begin{array}{c}N_{x}+K+q-2 \\ q\end{array}\right), M K\right)$, $K \in \llbracket 1, N_{X} \rrbracket$.

This expression may also be proved via an upper and a lower bound, but of the Frobenius norm of matrix $\tilde{\boldsymbol{R}}^{\circ q}$.

Lemma 3. (Upper Bound, general case)

$$
\left\|\tilde{\boldsymbol{R}}^{\circ q}\right\|_{F}^{2} \leq \sum_{m=1}^{M}\left(\left|\theta_{0}^{m, m}\right|^{2 q}-\theta_{\max }^{2 q}\right) \boldsymbol{w}^{T} \boldsymbol{w}+M^{2} \theta_{\max }^{2 q} .
$$

Proof. Again, the proof is really similar to [9, Lemma 1].

Lemma 4. (Lower bound, general case)

$$
\left\|\tilde{\boldsymbol{R}}^{\circ q}\right\|_{F}^{2} \geq \frac{\left|\sum_{m=1}^{M} \theta_{0}^{m, m}\right|^{2}}{l_{N_{x}, N_{y}, K, q}}
$$


with $l_{N_{x}, N_{y}, K, q}=\min \left(\left(\begin{array}{c}N_{X}+q-1 \\ q\end{array}\right),\left(\begin{array}{c}N_{x}+K+q-2 \\ q\end{array}\right), M K\right)$.

Proof. In the case where $q \neq 1$, some studies have been done (see for instance [27], [28, Section 1.2] or [29, Section 4]) in order to get an upper bound of $\operatorname{rank}\left(\tilde{\boldsymbol{R}}^{\circ q}\right)$ from an upper bound of $\operatorname{rank}(\tilde{\boldsymbol{R}})$. The latter has already been considered in Appendix A:

$$
\operatorname{rank}(\tilde{\boldsymbol{R}}) \leq \min \left(M K, N_{x}+K-1, N_{x}+N_{y}-1\right) .
$$

Incidentally, it is possible to show that - more details are given in the following:

$$
\operatorname{rank}\left(\tilde{\boldsymbol{R}}^{\circ q}\right) \leq l_{N_{x}, N_{y}, K, q},
$$

with $l_{N_{x}, N_{y}, K, q}:=\min \left(\left(\begin{array}{c}N_{x}+q-1 \\ q\end{array}\right),\left(\begin{array}{c}N_{x}+K+q-2 \\ q\end{array}\right), M K\right)$. Finally, it should bring us to the conclusion that:

$$
\left\|\tilde{\boldsymbol{R}}^{\circ q}\right\|_{F}^{2} \geq \frac{\left|\sum_{m=1}^{M} \theta_{0}^{m, m}\right|^{2}}{l_{N_{x}, N_{y}, K, q}} .
$$

In order to be more thorough, let us give some details of (52). This equation is based on the following property.

Property 1. Let $\boldsymbol{R}$ be a square matrix of size $m \times m$ such that $\boldsymbol{R}=\boldsymbol{X} \boldsymbol{Y}^{H}$, where $\boldsymbol{X}, \boldsymbol{Y} \in \mathbb{C}^{m \times n}$. Denote the columns of both matrices by respectively $\boldsymbol{x}_{i}$ and $\boldsymbol{y}_{i}, i \in \llbracket 1, n \rrbracket$. The rank of matrix $\boldsymbol{R}^{\circ q}$ can be upper-bounded as:

$$
\operatorname{rank}\left(\boldsymbol{R}^{\circ q}\right) \leq\left(\begin{array}{c}
n+q-1 \\
q
\end{array}\right) .
$$

Proof of the property. Matrix $\boldsymbol{R}$ can be developed as :

$$
\boldsymbol{R}=\sum_{i=1}^{n} \boldsymbol{x}_{i} \boldsymbol{y}_{i}^{H}=\sum_{i=1}^{n} \boldsymbol{R}_{i},
$$

with $\boldsymbol{R}_{i}=\boldsymbol{x}_{i} \boldsymbol{y}_{i}^{H}$. Hence,

$$
\boldsymbol{R}^{\circ q}=\underbrace{\boldsymbol{R} \circ \cdots \circ \boldsymbol{R}}_{q}=\left(\sum_{i=1}^{n} \boldsymbol{R}_{i}\right)^{\circ q} .
$$

Applying the multinomial theorem [28] gives :

$$
\boldsymbol{R}^{\circ q}=\sum_{\sum_{i=1}^{n} q_{i}=q}\left(\begin{array}{c}
q \\
q_{1}, \ldots, q_{n}
\end{array}\right)\left(\boldsymbol{R}_{1}^{\circ q_{1}} \circ \cdots \circ \boldsymbol{R}_{n}^{\circ q_{n}}\right),
$$

where $\left(\begin{array}{c}q \\ q_{1}, \ldots, q_{n}\end{array}\right)=\frac{q !}{q_{1} ! \ldots q_{n} !}$ is a multinomial coefficient.

Using the following properties, defined for two matrices $\boldsymbol{A}$ and $\boldsymbol{B}$ :

$$
\begin{aligned}
& \operatorname{rank}(\boldsymbol{A}+\boldsymbol{B}) \leq \operatorname{rank}(\boldsymbol{A})+\operatorname{rank}(\boldsymbol{B}), \\
& \operatorname{rank}(\boldsymbol{A} \circ \boldsymbol{B}) \leq \operatorname{rank}(\boldsymbol{A}) \operatorname{rank}(\boldsymbol{B}), \\
& \operatorname{rank}(\boldsymbol{A B}) \leq \min (\operatorname{rank}(\boldsymbol{A}), \operatorname{rank}(\boldsymbol{B})),
\end{aligned}
$$

it comes that :

$$
\begin{aligned}
\operatorname{rank}\left(\boldsymbol{R}^{\circ q}\right) & \leq \sum_{\sum_{i=1}^{n} q_{i}=q} \operatorname{rank}\left(\boldsymbol{R}_{1}\right)^{q_{1}} \times \cdots \times \operatorname{rank}\left(\boldsymbol{R}_{n}\right)^{q_{n}} \\
& \leq \sum_{\sum_{i=1}^{n} q_{i}=q} 1,
\end{aligned}
$$

because $\operatorname{rank}\left(\boldsymbol{R}_{i}\right) \leq 1$.
But the cardinality of set $\left\{q_{1}, \ldots, q_{n} \mid \sum_{i=1}^{n} q_{i}=q\right\}$ is equal to the number of $q$-combinations from a set of $n$ elements. It thus means that :

$$
\operatorname{rank}\left(\boldsymbol{R}^{\circ q}\right) \leq\left(\begin{array}{c}
n+q-1 \\
q
\end{array}\right) .
$$

Besides, as $\boldsymbol{R}^{\circ q}$ is a square matrix of order $m$, it follows :

$$
\operatorname{rank}\left(\boldsymbol{R}^{\circ q}\right) \leq \min \left(m,\left(\begin{array}{c}
n+q-1 \\
q
\end{array}\right)\right) .
$$

In particular, if $\operatorname{rank}(\boldsymbol{R}) \leq k$, there exists matrices $\boldsymbol{A}$ and $\boldsymbol{B}$ of size $m \times k$ such that $\boldsymbol{R}=\boldsymbol{A} \boldsymbol{B}^{H}$, so that :

$$
\operatorname{rank}\left(\boldsymbol{R}^{\circ q}\right) \leq \min \left(m,\left(\begin{array}{c}
k+q-1 \\
q
\end{array}\right)\right) .
$$

From the defintion of matrix $\tilde{\boldsymbol{R}}^{\circ q}$, Eq. (52) may be obtained straightforwardly.

\section{ACKNOWLEDGMENT}

This work was funded by Thales Land \& Air Systems and partly supported by a DGA-MRIS scholarship.

\section{REFERENCES}

[1] M. Pursley, "Performance evaluation for phase-coded spread-spectrum multiple-access communication - part i: System analysis," IEEE Transactions on Communications, vol. 25, no. 8, pp. 795-799, August 1977.

[2] J. Li and P. Stoica, MIMO Radar Signal Processing. Wiley, 2009.

[3] H. Deng, "Polyphase code design for orthogonal netted radar systems," IEEE Transactions on Signal Processing, vol. 52, no. 11, pp. 3126-3135, Nov 2004.

[4] J. Song, P. Babu, and D. P. Palomar, "Optimization methods for designing sequences with low autocorrelation sidelobes," IEEE Transactions on Signal Processing, vol. 63, no. 15, pp. 3998-4009, Aug 2015.

[5] C. Ding and T. Feng, "A Generic Construction of Complex Codebooks Meeting the Welch Bound," IEEE Transactions on Information Theory, vol. 53, no. 11, pp. 4245-4250, Nov 2007.

[6] C. Xiang, C. Ding, and S. Mesnager, "Optimal codebooks from binary codes meeting the levenshtein bound," IEEE Transactions on Information Theory, vol. 61, no. 12, pp. 6526-6535, Dec 2015.

[7] L. Welch, "Lower bounds on the maximum cross-correlation of signals (Corresp.)," IEEE Trans. Inf. Theory, vol. 20, no. 3, pp. 397-399, 1974.

[8] V. Levenshtein, "New lower bounds on aperiodic crosscorrelation of binary codes," in Proceedings of the IEEE International Symposium on Information Theory, 1998, p. 471.

[9] - "New lower bounds on aperiodic crosscorrelation of binary codes," IEEE Trans. Inf. Theory, vol. 45, no. 1, pp. 284-288, 1999.

[10] Z. Liu, Y. L. Guan, U. Parampalli, and S. Boztas,, "Quadratic weight vector for tighter aperiodic levenshtein bound," in Information Theory Proceedings (ISIT), 2013 IEEE International Symposium on, July 2013, pp. 3130-3134

[11] F. Arlery, U. Tan, and O. Rabaste, "Generalization and Improvement of the Levenshtein Lower Bound for Aperiodic Correlation," IEEE Transactions on Information Theory, vol. 65, no. 2, pp. 1258-1266, Feb 2019.

[12] Z. Liu, U. Parampalli, Y. Guan, and S. Boztaş, "A new weight vector for a tighter Levenshtein bound on aperiodic correlation," IEEE Trans. Inf. Theory, vol. 60, no. 2, pp. 1356-1366, 2014.

[13] O. Rabaste and L. Savy, "Mismatched filter optimization for radar applications using quadratically constrained quadratic programs," IEEE Transactions on Aerospace and Electronic Systems, vol. 51, no. 4, pp. 3107-3122, 2015.

[14] P. Stoica, J. Li, and M. Xue, "Transmit codes and receive filters for radar," IEEE Signal Processing Magazine, vol. 25, no. 6, pp. 94-109, 2008.

[15] J. Li, P. Stoica, and X. Zheng, "Signal synthesis and receiver design for mimo radar imaging," IEEE Transactions on Signal Processing, vol. 56, no. 8, pp. 3959-3968, 2008. 
[16] H. Deng, "Polyphase Code Design for Orthogonal Netted Radar Systems," IEEE Transactions on Signal Processing, vol. 52, no. 11, pp. 3126-3135, Nov 2004.

[17] M. Rihan and L. Huang, "Optimum co-design of spectrum sharing between mimo radar and mimo communication systems: An interference alignment approach," IEEE Transactions on Vehicular Technology, vol. 67 , no. 12, pp. 11667-11680, 2018.

[18] T. Aittomki and V. Koivunen, "Mismatched filtering for non-cooperative radar spectrum sharing," in 2019 53rd Asilomar Conference on Signals, Systems, and Computers, 2019, pp. 550-553.

[19] Q. Li, K. Dai, Y. Zhang, and H. Zhang, "Integrated waveform for a joint radar-communication system with high-speed transmission," IEEE Wireless Communications Letters, vol. 8, no. 4, pp. 1208-1211, 2019.

[20] R. Gold, "Optimal Binary Sequences for Spread Spectrum Multiplexing (Corresp.)," IEEE Transactions on Information Theory, vol. 13, no. 4, pp. 619-621, October 1967.

[21] J. M. Baden, "Efficient Optimization of the Merit Factor of Long Binary Sequences," IEEE Transactions on Information Theory, vol. 57, no. 12, pp. 8084-8094, Dec 2011.

[22] M. Soltanalian, B. Tang, J. Li, and P. Stoica, "Joint Design of the Receive Filter and Transmit Sequence for Active Sensing," IEEE Signal Processing Letters, vol. 20, no. 5, pp. 423-426, May 2013.

[23] L. Xu, H. Liu, K. Yin, H. Zang, S. Zhou, and H. Wang, "Joint Design of Phase Coded Waveform and Mismatched Filter," in 2015 IEEE Radar Conference, Oct 2015, pp. 32-36.

[24] U. Tan, O. Rabaste, C. Adnet, and J.-P. Ovarlez, "A Sequence-Filter Joint Optimization," in 26th European Signal Processing Conference (EUSIPCO 2018), September 2018, pp. 1-5.

[25] Z. Liu, Y. L. Guan, and W. H. Mow, "Asymptotically Locally Optimal Weight Vector Design for a Tighter Correlation Lower Bound of Quasi-Complementary Sequence Sets," IEEE Transactions on Signal Processing, vol. 65, no. 12, pp. 3107-3119, 2017.

[26] S. Boztaş, "New Lower Bounds on the Periodic Crosscorrelation of QAM Codes with Arbitrary Energy," in Applied Algebra, Algebraic Algorithms and Error-Correcting Codes (AAECC), 13th International Symposium on, vol. 1719, 11 1999, pp. 410-419.

[27] I. Peng and S. Waldron, "Signed frames and hadamard products of gram matrices," Linear Algebra and its Applications, vol. 347, no. 1, pp. 131 - 157, 2002.

[28] R. P. Stanley, Enumerative Combinatorics: Volume 1, 2nd ed. New York, NY, USA: Cambridge University Press, 2011.

[29] J. Calmet, W. Geiselmann, and J. Mller-Quade, Mathematical Methods in Computer Science: Essays in Memory of Thomas Beth, 1st ed. Springer Publishing Company, Incorporated, 2008.

Uy Hour Tan received the Master of Applied Mathematics in 2014 from Sorbonne Université - former Université Pierre et Marie Curie - Paris, France, and the Ph.D. degree in Signal Processing in 2019 from CentraleSupélec, Université Paris-Saclay, France, in partnership with Thales Land \& Air Systems and ONERA. He is currently research engineer at Thales. His research interests include optimisation methods, waveform design and MIMO radar.

Fabien Arlery received the Engineering degree in Electronics in 2013 from the École Nationale Supérieure d'Électronique, Informatique, Télécommunications, Mathématique et Mécanique de Bordeaux (ENSEIRB-MATMECA), Talence, France, and the Ph.D. degree in Signal Processing in 2017 from the École Nationale Supérieure des Télécommunications d'Évry (Telecom SudParis, Université Paris-Saclay, Évry, France). His main area of research has been on waveform design and their performance assessment for radar applications. He is currently research engineer at Thales.
Olivier Rabaste received the Engineering degree in Telecommunications in 2003 from the Ecole Nationale Supérieure des Télécommunications de Bretagne, Brest, France, the M.S. degree in digital communications systems and technologies with distinction in 2003 from the Chalmers University of Technology, Göteborg, Sweden, and the Ph.D. degree in 2006 from the Ecole Nationale Supérieure des Télécommunications de Bretagne. He is currently research engineer at ONERA, the French Aerospace Lab since 2008. His research interests are on detection, estimation, tracking and waveform design for radar applications such as surveillance, MIMO and passive radar.

Frédéric Lehmann received the E.E. degree and the M.S.E.E. degree from ENSERG, Grenoble, France, in 1998, and the Ph.D. degree in electrical engineering from the National Polytechnical Institute, Grenoble, France. He worked as a Research Engineer with STMicroelectronics from 1999 to 2002. From 2003 to 2004, he was a Post-Doctoral Researcher at the Laboratory for Analysis and Architecture of Systems, CNRS, Toulouse, France. He is currently a Professor with Telecom SudParis, Institut Polytechnique de Paris. His main research interests are in the area of communication theory, nonlinear signal processing, and statistical image processing.

Jean-Philippe Ovarlez (M'06) was born in Denain, France in 1963. He received jointly the engineering degree from Ecole Supérieure d'Electronique Automatique et Informatique (ESIEA), Paris, France and the Diplôme d'Etudes Approfondies degree in Signal Processing from University of Paris XI, Orsay, France and the Ph.D. degree in Physics from the University of Paris VI, Paris, France, in 1987 and 1992, respectively. In 2011, he obtained a Research Directorship Habilitation (HDR) thesis in Signal Processing from the University of Paris-Sud and his qualification to the University Professor position. In 1992, he joined the Electromagnetic and Radar Division of the French Aerospace Lab (ONERA), Palaiseau, France, where he is currently Director of Research and member of the Scientific Committee of the ONERA Physics domain. Since 2008, he is attached at a part time to CentraleSupélec SONDRA Lab, in charge of Signal Processing activities supervision. In 2015, he becomes member of Special Area Team (SAT) in Theoretical and Methodological Trends in Signal Processing (TMTSP), EURASIP and treasurer of the IEEE GRSS French Chapter in 2016. His research interests are centered in the topic of Statistical Signal Processing for radar and SAR applications such as Time-Frequency, imaging, detection and parameters estimation. 